\title{
ANTIBIOTIC SUSCEPTIBILITY PATTERN OF KLEBSIELLA PNEUMONIAE ISOLATED FROM CASES OF URINARY TRACT INFECTION IN A TERTIARY CARE SETUP
}

\author{
Anila Varghese1, Shareen George2, Radhakuttyamma Gopalakrishnan ${ }^{3}$, Anna Mathew ${ }^{4}$ \\ ${ }^{1}$ Final year MBBS Student, M. O. S. C. Medical College, Kolenchery. \\ ${ }^{2}$ Associate Professor, Department of Microbiology, M. O. S. C. Medical College, Kolenchery. \\ 3 Professor and HOD, Department of Microbiology, M. O. S. C. Medical College, Kolenchery. \\ ${ }^{4}$ Professor, Department of Pharmacology, M. O. S. C. Medical College, Kolenchery.
}

\begin{abstract}
Urinary Tract Infection (UTI) is second on the ranking of most common infection in community practice. It leads to significant morbidity and a high economic burden for treatment. Klebsiella pneumoniae accounts for $2^{\text {nd }}$ highest organism isolated from urine samples of UTI patients after Escherichia coli. The management of UTI is complicated by the increasing prevalence of antibiotic resistant strains of Klebsiella pneumonia. Therefore, knowledge of the antibiotic resistance patterns of the pathogen is important not only to provide an appropriate therapy, but also for the prevention of resistance amongst the microbe.
\end{abstract}

\section{OBJECTIVE}

The present study was therefore undertaken to determine the antibiotic susceptibility pattern of Klebsiella pneumonia causing UTI in patients admitted to a tertiary care hospital.

\section{MATERIAL AND METHODS}

The details of Klebsiella pneumonia grown in urine samples received in the Department of Microbiology, MOSC Medical College, were collected from the laboratory registers. These urine samples were then processed using standard methods and antibiotic susceptibility testing was done by Kirby Bauer's disc diffusion method.

\section{RESULT}

During the period of 4 months, 35 urine samples yielding Klebsiella pneumonia were processed. These strains showed $100 \%$ resistance to Ampicillin, around 70-85\% resistance to first, second and third generation Cephalosporins. They showed maximum sensitivity to Imipenem (74.3\%), followed by Colistin (77\%), Amikacin (65.7\%), Meropenem (65.7\%) and Piperacillin-Tazobactam (65.7\%).

\section{CONCLUSION}

In our study, the high rate of resistance to routinely prescribed drugs like Co-trimoxazole, Norfloxacin and Nitrofurantoin could be attributed to the frequent use of these antibiotics. Carbapenems (Imipenem or Meropenem) and Amikacin should be considered as reserved drugs, especially for nosocomial infections.

\section{KEYWORDS}

Klebsiella Pneumonia, Urinary Tract Infections (UTI), Antibiotic Resistance.

HOW TO CITE THIS ARTICLE: Varghese A, George S, Gopalakrishnan R, et al. Antibiotic susceptibility pattern of Klebsiella pneumoniae isolated from cases of urinary tract infection in a tertiary care setup. J. Evolution Med. Dent. Sci. 2016;5(29):

1470-1474, DOI: $10.14260 /$ jemds/2016/346

\section{INTRODUCTION}

Urinary Tract Infections (UTIs) are amongst the most common infections encountered in clinical practice.(1) Urinary infections cause fewer complications than nosocomial infections, but they occasionally can cause bacteraemia and death.(2) Urinary Tract Infection (UTI) can be caused by Gramnegative bacteria such as Escherichia coli, Klebsiella pneumoniae, Enterobacter species, Proteus species and gram-positive bacteria like Enterococcus species and Staphylococcus saprophyticus. (3) Excessive and/or inappropriate use of antibiotics in treating UTIs is responsible for the emergence and spread of multi-drug resistant urinary bacterial pathogens.

Financial or Other, Competing Interest: None.

Submission 20-02-2016, Peer Review 15-03-2016,

Acceptance 22-03-2016, Published 09-04-2016.

Corresponding Author:

Dr. Shareen George,

Associate Professor

Department of Microbiology,

M.O.S.C. Medical College, Kolenchery.

E-mail: shareendushyanth@gmail.com

DOI: $10.14260 / \mathrm{jemds} / 2016 / 346$
Klebsiella pneumoniae is the second most frequently isolated species from UTI, after Escherichia coli.(4) The ability of the bacteria to adhere to host structures is considered essential for the development of infection. $(5,6)$ UTI usually affects the lower urinary tract, but sometimes both the lower and upper urinary tracts can be involved. The term cystitis has been used to define the lower UTI and is characterized by symptoms such as dysuria, frequency, urgency and suprapubic tenderness. The presence of the lower UTI symptoms does not exclude the upper UTI, which is often present in most UTI cases. (3)

The treatment of UTI can be classified into uncomplicated and complicated on the basis of their choice of treatment.(7) The treatment of UTIs varies according to the age of the patient, sex, underlying disease, infecting agent and whether there is lower or upper urinary tract involvement.(8) Empirical antibiotic therapy is usually applied. Urinary pathogens have shown a changing pattern of susceptibility to antibiotics resulting in an increase in resistance to commonly used antibiotics. Of particular concern is the recent appearance of Klebsiella pneumoniae strains that produce the enzymes, Extended Spectrum Beta Lactamases (ESBLs). Production of ESBL is frequently plasmid encoded and bears 
clinical significance. Plasmids responsible for ESBL production frequently carry genes encoding resistance to other drug classes also. Therefore, antibiotic options in the treatment of ESBL producing organisms are extremely limited.(9) Detection of ESBL production is important. One major concern is the spread of ESBL positive bacteria within hospitals, which may lead to outbreaks or to endemic occurrence.

Years of antibiotic over-prescription and abuse on one hand and a decline in the development of novel antibiotics on the other have led to a tendency among physicians to shy away from prompt and aggressive prescription of the most commonly used drugs. $(10,11)$ This is especially the case with broad-spectrum antibiotics. The predicted changes in the pathogens and their occurrence makes it highly advisable that empirical, first-line antibiotic treatment should be reviewed periodically in every regional tertiary medical centre. (8,12-13) Due to rising antibiotic resistance among uropathogens, it is important to have local hospital-based knowledge of the organisms causing UTI and their antibiotic sensitivity patterns.(14) It is of great importance for the institution to know the local antibiotic resistance patterns in order to implement suitable infection control measures and develop a rational antibiotic policy with local recommendations for antibiotic use. These surveillance data are also used to assess the effectiveness of the measures taken and to identify new points for intervention to control bacterial resistance.

The present study was therefore undertaken to assess the current antibiotic resistance pattern in the Klebsiella pneumoniae species isolated in a tertiary care hospital in Kerala.

\section{MATERIALS AND METHODS}

This cross-sectional study was undertaken in the Department of Microbiology, MOSC Medical College, Kolenchery, India. This study was undertaken as part of the STS ICMR (Short Term Studentship-Indian Council of Medical Research) Project and done on urine samples from patients admitted to the MOSC Medical Mission Hospital during the period from June to September 2014. Only the initial sample of an individual received was included to avoid duplication. The exclusion criteria for the study were suprapubic aspirates, repeat samples from the same patients and urine samples mixed with other uropathogens along with Klebsiella pneumonia. The samples collected were processed as per standard methods. The study protocol was approved by the Ethics Committee of the Institute.

The urine samples collected from the patients were processed according to the standard microbiological procedures with a calibrated one microlitre loop using the semi-quantitative method of plating onto Cysteine Lactose Electrolyte Deficient (CLED) medium and blood agar medium (Hi-media, Mumbai, India) to isolate the uropathogens. Inoculated culture plates were kept in the incubator at $37^{\circ} \mathrm{C}$ for 24 hours. All the bacteria were identified using morphological, microscopy and biochemical tests following standard procedures described by Cowan and Steel (1974) and Cheesborough (2006).(15,16)

The antibiotic sensitivity testing was performed using the Kirby-Bauer method as per the Clinical Laboratory Standards Institute (CLSI).(17,18) The isolates were tested for
Ampicillin $(10 \mu \mathrm{g})$, Cotrimoxazole $(25 \mu \mathrm{g})$, Amoxiclav (20/10 $\mu \mathrm{g})$, Gentamicin $(10 \mu \mathrm{g})$, Cephalexin $(30 \mu \mathrm{g})$, Cefuroxime (30 $\mu \mathrm{g})$, Cefotaxime $(30 \mu \mathrm{g})$, Ceftazidime $(30 \mu \mathrm{g})$, Ciprofloxacin (5 $\mu \mathrm{g})$ and Amikacin $(30 \mu \mathrm{g})$ in $(10 \mu \mathrm{g})$, Ceftriaxone $(30 \mu \mathrm{g})$ Norfloxacin $(10 \mu \mathrm{g})$, Nitrofurantoin $(300 \mu \mathrm{g})$, Cefepime (30 $\mu \mathrm{g})$, Colistin $(10 \mu \mathrm{g})$, Meropenem $(10 \mu \mathrm{g})$, Nalidixic acid (30 $\mu \mathrm{g})$, Piperacillin $(100 \mu \mathrm{g})$, Piperacillin-Tazobactam $(100 / 10$ $\mu \mathrm{g})$, Ceftazidime-clavulanic acid combination $(30 / 10 \mu \mathrm{g} / \mathrm{disc})$ and Imipenem $(10 \mu \mathrm{g})($ Hi-media, Mumbai).

\section{Extended Spectrum $\beta$-Lactamase Detection}

The screening for Extended Spectrum Beta Lactamase (ESBL) was done using Ceftazidime $(\leq 22 \mathrm{~mm})$, Cefotaxime $(\leq 27 \mathrm{~mm})$, and Ceftriaxone ( $\leq 25 \mathrm{~mm}$ ). If the organisms showed a zone of inhibition lower than the minimum for any antibiotic disc, ESBL positivity was suspected. The phenotypic confirmation was done by testing the strain against Ceftazidime (Ca) and Ceftazidime-clavulanic acid combination $(30 / 10 \mathrm{mcg} /$ discs $)$. In this test, an overnight culture suspension of the bacterial isolate was adjusted to 0.5 McFarland's standard. Lawn culture was done on the Mueller-Hinton Agar (MHA) plate. The Ceftazidime and Ceftazidime-clavulanic acid discs were placed $20 \mathrm{~mm}$ apart on the agar surface. After incubating at $37^{\circ} \mathrm{C}$ for 24 hours, $\mathrm{a} \geq 5 \mathrm{~mm}$ increase in the zone diameter in comparison to ceftazidime was considered indicative of ESBL production. Escherichia coli ATCC 25922 was used as an ESBL-negative and Klebsiella pneumoniae 700603 was used as an ESBL-positive reference strain.(19)

\section{RESULT}

During the 4-month period, a total of 2202 urine samples were processed for culture and sensitivity testing. Of the total urine samples studied, $92(4.1 \%)$ showed mixed bacterial growth may be due to improper specimen collection or delay in transporting the sample to the laboratory. A total of 702 different organisms were isolated, thus the culture positivity was $32 \%$. Out of the total 702 different organisms, E. coli was the most isolated organism accounting for 336 (48\%). The second commonest isolate was Klebsiella pneumoniae which accounted for 117 (17\%) followed by Candida species (10\%), Streptococcus species (6\%), Citrobacter species (4.3\%), Enterobacter species (4\%), Staphylococcus species $(3.7 \%)$, Pseudomonas aeruginosa (3.5\%), Acinetobacter species (2.2\%) and other Klebsiella species (2\%) (Table no. 1).

As this study was part of the STS ICMR Project, only 35 urine samples positive for Klebsiella pneumonia were selected; $60 \%$ out of these 35 samples were from patients in the age group 41-80 years (Figure no. 1). Of the 35 urine samples studied, 23 (66\%) were from females and 12 (34\%) were from males (Figure no. 2); $86 \%$ of the tested urine samples were midstream samples and $14 \%$ from catheterized patients (Figure no. 3). All the catheterized patients were inpatients. Only $57 \%$ of the patients included in the study had symptoms of UTI-dysuria, frequency of micturition, lower abdominal pain, vomiting and burning sensation during micturition. The rest $43 \%$ of patients were admitted or came to the casualty with complaints other than UTI. But when routine urine microscopy was done, pus cells were found to be very high and hence urine was sent for culture and sensitivity.

Klebsiella pneumoniae isolated from the urine samples in the study were most resistant to Ampicillin (100\%) 
followed by Cefuroxime (85.7\%) and Cephalexin (80\%). Carbapenems-Imipenem (85.7\%) and Meropenem (65.7\%) along with Colistin (70\%) and Amikacin (65.7\%) were highly sensitive (Figure no. 4). Out of the 35 samples processed, 12 $(34.2 \%)$ were ESBL producers.

\begin{tabular}{|c|c|c|}
\hline Organisms & $\begin{array}{c}\text { Total } \\
\text { No. }\end{array}$ & Percentage (\%) \\
\hline E. coli & 336 & 48 \\
\hline Klebsiella pneumoniae & 117 & 17 \\
\hline Candida species & 68 & 10 \\
\hline Streptococcus species & 40 & 6 \\
\hline Citrobacter species & 30 & 4.3 \\
\hline Enterobacter species & 29 & 4 \\
\hline Staphylococcus species & 26 & 3.7 \\
\hline Pseudomonas aeruginosa & 25 & 3.5 \\
\hline Acinetobacter species & 16 & 2.2 \\
\hline Klebsiella species & 15 & 2 \\
\hline \multicolumn{2}{|c|}{ Table 1: Organisms Isolated in the Study Group } \\
\hline
\end{tabular}

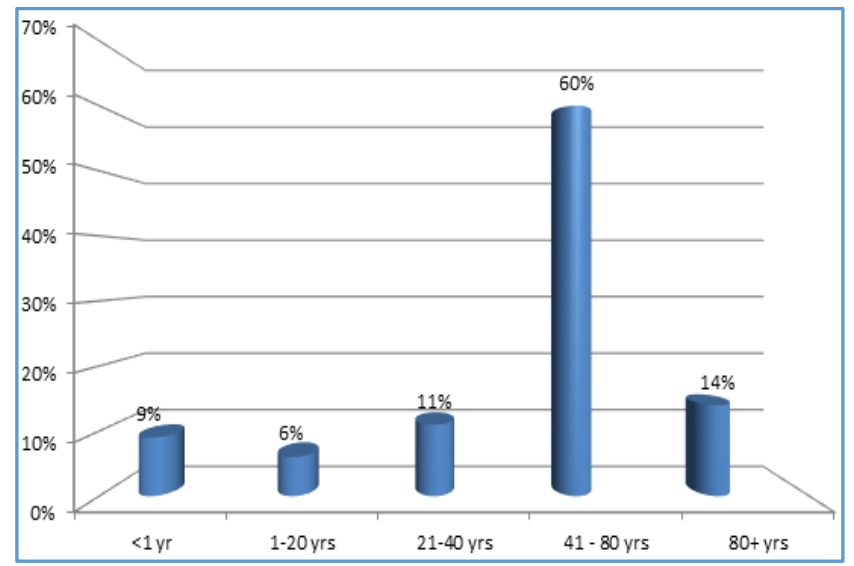

Fig. 1: Age Wise Distribution of Patients with UTI

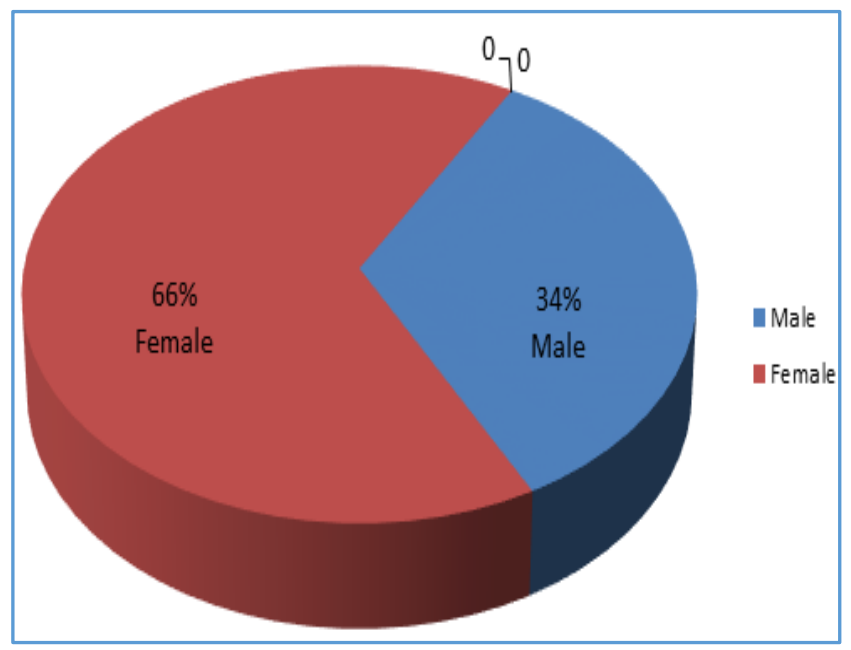

Fig. 2: Sex Wise Distribution of Patients with UTI

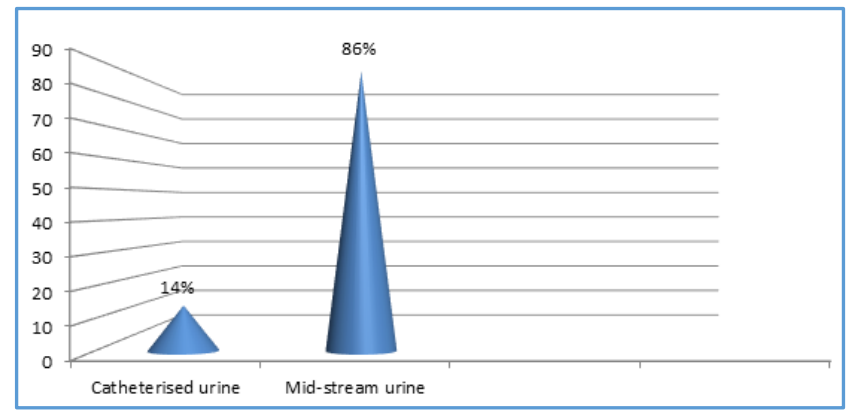

Fig. 3: Percentage of Type of Urine Sample

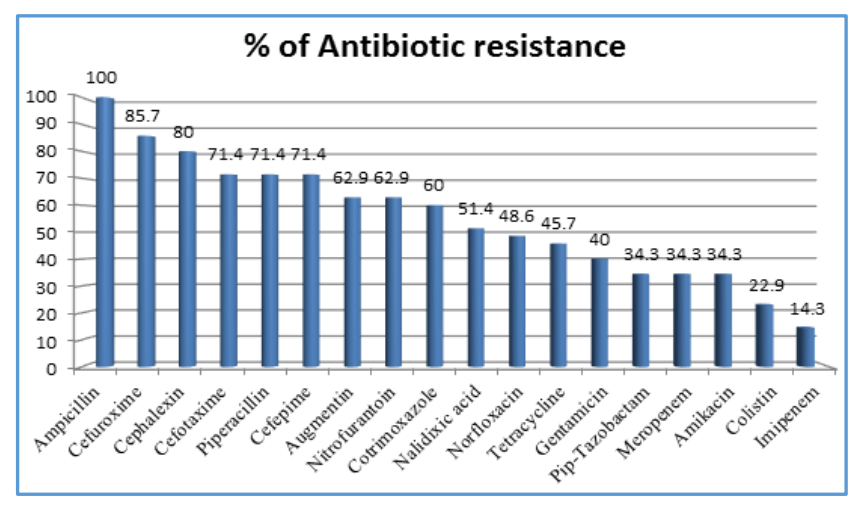

Fig. 4: Percentage of Antibiotic Resistance Pattern

\section{DISCUSSION}

This study was done to determine the antibiotic susceptibility patterns of Klebsiella pneumonia isolated from urine samples in a tertiary care centre in Kerala.

$23(66 \%)$ of the urine samples were from females and $12(34 \%)$ were from males. This is consistent with other studies.(20-24) UTI is more common in females than in males, as female urethra structurally found less effective for preventing the bacterial entry.(25) It may be due to the proximity of the genital tract and urethra and adherence of urothelial mucosa to the mucopolysaccharide lining.(26) The other main factors which make females more prone to UTI are pregnancy and sexual activity. Sexual activity in females increases the risk of urethra contamination as the bacteria could be pushed into the urethra during sexual intercourse as well as bacteria being massaged up the urethra into the bladder during child birth.

The age wise data was analysed in order to assess the frequency of UTI in different age groups. This study portrayed that people in the age group $41-80$ years $(60 \%)$ were more prone to UTI than any other groups. This result is in comprehension with other studies.(23, 27-29)

About 17\% (6) of the age group 41-80 years were males and $43 \%$ (15) were females. In older men, the incidence of UTI may increase due to the prostatic obstruction or subsequent instrumentation like Foley's catheter.(30) Only $57 \%$ of the patients included in the study had symptoms of UTI. The rest $43 \%$ of patients had come to the hospital with complaints other than UTI. Depending on the presence of pus cells in direct microscopy, the overall condition of the patient and the antibiotic susceptibility pattern, a holistic decision regarding treatment was taken by the Microbiologist and the Clinician.

Klebsiella pneumonia isolated from the 35 urine samples included in the study showed $100 \%$ resistance to 
Ampicillin. This is consistent with results of other studies, which have shown a resistance from $93 \%$.(31) to $98 \%$.(20)

In this study the resistance results were Amoxiclav (62.9\%), Amikacin (32.3\%), Nalidixic acid (51.4\%) and Norfloxacin (48.6\%). In a similar study, Somashekara SC et al. showed the antibiotic resistance pattern of Klebsiella pneumonia to be Amoxiclav (68\%), Amikacin (26\%), Nalidixic acid (48.2\%) and Norfloxacin (38\%).(21)

The three most commonly prescribed antibiotics for UTI are Co-trimoxazole, Norfloxacin and Nitrofurantoin. The resistance of Klebsiella pneumonia to these drugs were $60 \%$, $48.6 \%$ and $62.9 \%$ respectively. This increased resistance pattern can be attributed to the frequent use of these antibiotics, as a result of which the organism may have developed a different mode of action.

Overall resistance to various generations of Cephalosporins and Beta Lactams is very high on account of the production of Extended Spectrum Beta Lactamases (ESBLs). Over the past two decades, there has been a wide use of extended broad-spectrum antimicrobial agents to meet the emerging challenge of treating UTIs due to gram-negative bacilli. However, these microbes have developed multiple antimicrobial resistance mechanisms including enhanced drug efflux, alterations of the drug target and the production of plasmid-mediated $\beta$ lactamases.(32) Klebsiella pneumoniae isolates have in general high rates of resistance to the commonly used antimicrobial agents.

All Klebsiella pneumonia isolates are naturally resistant to Amoxicillin and Ampicillin, due to a constitutively expressed chromosomal class $\beta$ lactamase.(33) Co-trimoxazole, used extensively in general practice, was first introduced as a combination drug that inhibits bacterial production of folate, causing a bacteriostatic effect. The frequent use of this antimicrobial agent for the treatment of community-acquired UTIs has led to higher resistance levels. In our study, antimicrobial non-susceptibility to Co-trimoxazole (60\%) displayed by urinary Klebsiella pneumonia isolates was similar to that reported in Algeria (63.2\%).(34) The high incidence of ESBL-producing Klebsiella pneumonia can mainly be explained by spread of these multi-drug resistant strains like the class A ESBLs, TEM, SHV and CTX types.(22) ESBL producing Klebsiella pneumonia isolates is a wellrecognized problem and explains the high resistance rates to commonly used antimicrobial agents.

Due to this steady decrease in susceptibility to nonCarbapenem antibiotics over time increased Carbapenem consumption has been subsequently reported and speculated to be associated with an increasing resistance of Klebsiella pneumonia isolates to Carbapenems. These antimicrobial agents are considered the treatment of choice for serious infections caused by ESBL-producing and/or AmpC $\beta$ lactamase-producing Enterobacteriaceae because of their high stability to $\beta$ lactamase hydrolysis and the relatively high susceptibility of ESBL producers to carbapenems. (35)

Colistin has a sensitivity of $77 \%$ and moderate sensitivity of $8.6 \%$, but considering the risk versus benefits of this drug it is rarely prescribed.

The most useful antibiotic as per this study was Imipenem $(74.3 \%$ sensitivity and moderate sensitivity of $11.4 \%)$. This drug is relatively expensive when compared to most antibiotics frequently used. This has probably restricted its procurement and indiscriminate use, therefore making the organisms susceptible to it.

Hence, there is a need to emphasize the rational use of antimicrobials and strictly adhere to the concept of "reserve drugs" to minimize the misuse of available drugs. Carbapenems (Imipenem or Meropenem) and Amikacin should be considered as reserved drugs, especially for nosocomial infections.

\section{CONCLUSION}

Through this study conducted at MOSC Medical College, Kerala, it has been found that Klebsiella pneumoniae is extremely resistant to the $\beta$ lactam group of drugs and cephalosporins. Amikacin and Carbapenems like Imipenem and Meropenem were the most sensitive antibiotics and hence they should be kept as reserved drugs. Since the antibiotic susceptibility patterns can vary, it is important to be updated so as to prescribe the most efficient drug for the patient, especially in conditions where empirical therapy is unavoidable. An effective antibiotic policy and draft guidelines should be introduced to preserve the effectiveness of antibiotics and for better patient management. Laboratory evidence of infection and antibiotic susceptibility testing should be carried out to help in the choice of drugs.

It is highly recommended that the clinician should become aware of the magnitude of the existing problem of antimicrobial resistance and help by rational prescription of antibiotics.

\section{ACKNOWLEDGEMENT}

The authors would like to thank Ms. Manju Jacob, Tutor, Department of Microbiology, for her skilled assistance, unflinching cooperation and support during the study. We also would like to thank all the laboratory technicians and clerical staff of the Department of Microbiology, MOSC Medical College, Kolenchery, for their consistent support and help in the study.

\section{REFERENCES}

1. Gatermann SG. Bacterial infections of the urinary tract. In: Borriello P, Murray PR, Funke G, editors. Topley \& Wilson's microbiology \& microbial infections, London: Hodder Arnold Publishers 10thed, 2007;vol III:p 671683.

2. Schappert SM. Ambulatory care visits to physician offices, hospital outpatient departments, and emergency departments: United States, 1997. Vital Health Stat 13, 1999;143(i-iv):1-39.

3. Sobel JD, Kaye D. Urinary tract infections. In: Mandell GL, Bennett JE, Dolin R, editors. Mandell, Douglas and Bennett's principles and practice of infectious diseases. Philadelphia, USA: Churchill Livingstone Elsevier publication 7thed, 2010;vol 1:p 958-972.

4. Stamm WE, McKevitt M, Roberts PL, et al. Natural history of recurrent urinary tract infections in women. Rev Infect Dis 1991;13(1):77-84.

5. Struve C, Bojer M, Krogfelt KA. Characterization of klebsiellapneumoniae type 1 fimbriae by detection of phase variation during colonization and infection and impact on virulence. Infect Immun 2008;76(9):40554065 . 
6. Lin $\mathrm{WH}$, Wang $\mathrm{MC}$, Tseng $\mathrm{CC}$, et al. Clinical and microbiological characteristics of klebsiella pneumonia isolates causing community-acquired urinary tract infections. Infection 2010;38(6):459-464.

7. Sabra SM, Abdel-Fattah MM. Epidemiological and microbiological profile of nosocomial infection in taif hospitals, KSA (2010-2011). World Journal of Medical Sciences 2012;7(1):1-9.

8. Warren JV, Abrutyn E, Hebel R, et al. Guidelines for the treatment of uncomplicated acute bacterial cystitis and acute pyelonephritis in women. Clin Infect Dis 1999;29(4):745-758.

9. Paterson DL, Bonomo RA. Extended spectrum beta lactamases: a clinical update. Clinical Microbiology Review 2005;18(4):657-686.

10. Gupta K, Hooton TM, Stamm WE. Increasing antimicrobial resistance and the management of uncomplicated community acquired urinary tract infections. Ann Intern Med 2001;135(1):41-50.

11. Manges AR, Johnson JR, Foxman B, et al. Widespread distribution of urinary tract infections caused by a multidrug-resistant escherichia coli clonal group. $\mathrm{N}$ Engl J Med 2001;345(14):1007-1013.

12. Colodner R, Keness Y, Chazan B, et al. Antimicrobial susceptibility of community-acquired uropathogens in northern Israel. Int J Antimicrob Agents 2001;18(2):189-192.

13. Huang ES, Stafford RS. National patterns in the treatment of urinary tract infections in women by ambulatory care physicians. Arch Intern Med 2002;162(1):41-47.

14. Kahlmeter G, ECO SENS. An international survey ofthe antimicrobial susceptibility of pathogens from uncomplicated urinary tract infections: the ECO SENS project. J Antimicrobial Chemother 2003;51(1):69-76.

15. Cheesborough M. Medical laboratory manual for tropical countries, microbiology (ELBS), New York, 2009; $2^{\text {nd }}$ edn:62-70.

16. Cowan SJ, Steel KJ. Cowan and steel manual for identification of medical bacteria, Cambridge university press, London, 1974;2nd edn.

17. Wayne PA. Performance standards for antimicrobial susceptibility testing, clinical and laboratory standards institute (CLSI), 20th information supplement M100-S20. USA, 2010.

18. Bauer AW, Kirby WM, Sherris JC, et al. Antibiotic susceptibily testing by a standardized single disc method. An J Clin Pathol 1966;45(4):493-496.

19. Wayne PA. Performance standards for antimicrobial susceptibility testing. Clinical and laboratory standards institute. 16th informational supplement M100-S16. 2006.

20. Somashekara SC, Deepalaxmi S, Jagannath N, et al. Retrospective analysis of antibiotic resistance pattern to urinary pathogens in a tertiary care hospital in south India. Journal of Basic Clinical Pharmacy 2014;5(4):105108.

21. Arul Prakasam KC, Kumar KGD, Vijayan M. A cross sectional study on distribution of urinary tract infection and their antibiotic utilization pattern in Kerala. International Journal of Research in Pharmaceutical and Biomedical Sciences 2012;4(3):1309-1316.
22. Bush K. New beta-lactamases in gram negative bacteria: diversity and impact on the election of antimicrobial therapy. Clinical Infectious diseases 2001;32:10851099.

23. Sarathbabu R, Ramani TV, Rao KB, et al. Antibiotic susceptibility pattern of klebsiella pneumoniae isolated from sputum, urine and pus samples. IOSR Journal of Pharmacy and Biological Sciences 2012;1(2):22783008.

24. Manjunath GN, Prakash R, Annam V, et al. Changing trends in the spectrum of antimicrobial drug resistance pattern of uropathogens isolated from hospitals and community patients with urinary tract infections in Tumkur and Bangalore. Int J Biol Med Res 2011;2(2):504-507.

25. Warren JW, Tenney JH, Hoopes JM. A prospective microbiologic study of bacteriuria in patients with chronic indwelling urethral catheters. Journal of Infectious Diseases 1982;146(6):719-723.

26. Kolawole AS, Kolawole OM, Kandaki-Olukemi YT, et al. Prevalence of urinary tract infections (UTI) among patients attending dalhatuaraf specialist hospital, Lafia, Nasarawa state, Nigeria. International Journal of Medicinal Medical Sciences 2009;1(5):163-167.

27. Akram M, Shahid M, Khan A. Etiology and antibiotic resistancepatterns of community acquired urinary tract infections in JNMC hospital Aligarh, India. Annals of Clinical Microbiology and Antimicrobials 2007;6(4):1-7.

28. Goettsch W, van Pelt W, Nagelkerke N, et al. Increasing resistance to fluoroquinolones in escherichia coli from urinary tract infection in the Netherlands. J Antimicrob Chemother 2000;46(2):223-228.

29. Anbumani N, Mallika M. Antibiotic resistance pattern in uropathogens in a tertiary care hospital. Indian Journal for the Practising Doctor 2007;4(1).

30. Jung-Sheng Y, Kun-Hung S, Wen-Chi C, et al. Effects of electro-acupuncture on benign prostate hyperplasia patients with lower urinary tract symptoms: a singleblinded, randomized controlled trial. Evidence-Based Complementary and Alternative Medicine 2011;Article ID 303198:pp 8.

31. Helio S Sader, Ronald N Jones, Patricia L Winokur, et al. Antimicrobial susceptibility of bacteria causing urinary tract infections in Latin American hospitals: results from the SENTRY antimicrobial surveillance program (1997). Clin Microbiol Infect 1999;5(8):478-487.

32. Tenover FC. Mechanisms of antimicrobial resistance in bacteria. Am J Med 2006;119(6 Suppl 1):S3-10, discussion S62-70.

33. Livermore DM. Beta-lactamases in laboratory and clinical resistance. Clin Microbiol 1995;8(4):557-584.

34. Bouzenoune F, Boudersa F, Bensaad A, et al. Les infections urinaires à Ain M'lila (Algérie). Résistance aux antibiotiques des 239 souchesisolées entre 2006 et 2007. Med Mal Infectieuses 2009;39(2):142-143.

35. Colodner R, Raz R, Chazan B, et al. Susceptibility pattern of ESBL-producing bacteria isolated from inpatients to five antimicrobial drugs in a community hospital in northern Israel. Int J Antimicrob Agents 2004;24:409410. 